\title{
Resistance to extinction as a function of sequence of events in partial reinforcement ${ }^{1}$
}

\author{
LAWRENCE S. MEYERS and E. J. \\ CAPALDI, Purdue University, Lafayette, \\ Ind. 47907
}

Two partial reinforcement groups, which differed in the type and frequency of run lengths experienced, were run in a Humphreys Board situation. These groups differed in extinction as predicted by sequential theory, and both were more resistant than a continuous reinforcement group. It was suggested that sequence learning may be independent of reinforcement contingencies.

Although early studies of the partial reinforcement effect (PRE) employed human Ss (e.g., Humphreys, 1939a, b), subsequent work has concentrated mainly on animal Ss. Sequential theory (e.g., Capaldi, 1966), for instance, employing animal data exclusively has identified number of $\mathrm{N}-\mathrm{R}$ transitions and N-length as important variables regulating the PRE. Investigations employing human Ss, however, have also implicated sequential variables. Restle $(1961,1966)$, for example, has suggested that both run length (number of $x$ events preceding a $y$ event) and the number of different run lengths strongly influence the decisions of an S. More recently, Gambino \& Myers (1967) have suggested that a model they developed for run structure was compatible with sequential theory.

The present study represents a further attempt to examine the operation of certain sequential variables in human $S$ s. Grosslight and his associates (Grosslight \& Child, 1947; Grosslight, Hall, \& Murnin, 1953) have shown that increasing the number of $\mathrm{N}-\mathrm{R}$ transitions will increase resistance to extinction (RE); consequently, the partial reinforcement (PR) groups in this study were equated on this variable as well as on percentage. The groups differed, however, in the number of experiences with the longer run length. A continuous reinforcement group was also included.

\section{SUBJECTS}

Thirty introductory psychology students, 9 males and 21 females, distributed randomly among the three groups $(N=10)$, participated in the study. APPARATUS

The apparatus, a modified Humphreys Board, $18 \%$ in. high and $20 \mathrm{in}$. wide, was mounted on plywood so that it was supported in an upright position. The entire right half of the board was painted dark green, the entire left half, orange. Each side contained two lights, their colors matching that of the background, and were placed as follows: Five inches down from the top and horizontally centered within the color was a $28-\mathrm{V}$ bulb; 5 in. directly below was a second smaller light of the same color. These lights were operated from behind the board by the throwing of two-pole mercury (silent) switches. The apparatus was placed on a desk and, together with an unpainted, upright piece of $19 \times 27$ in. plywood, effectively blocked $S$ from seeing $E$ during the actual running. For this experiment, $S$ received training on only one side of the apparatus, with color being counterbalanced.

DESIGN

Group C was given 12 "t" trials, in which the light came on each time after $S$ responded independent of S's guess. Group M received 29 trials containing a mixture of + and "-" (light failing to come on) trials, using the following schedule: + $--+-+----++-+--++--$ $+-+-\ldots++$. Group $F$ was run on the 29-trial sequence +-+---++-+++----+-+----+-++ . Note that for each of the latter two groups percentage of + trials is equated (about $42 \%$ ) with the number of + trials matching that of Group C. The PR groups were also equated on number of,-++- , and ++ transitions. They differ with regard to the type and frequency of run lengths of the event. Group $M$ (many run lengths) received run lengths of $1,2,3$, and 4 , at frequencies of $3,2,2$, and 1 , respectively; Group F (few run lengths) received run lengths of 1 and 4 at frequencies of 5 and 3 , respectively.

\section{DEPENDENT VARIABLE}

An "expectancy" scale developed by James \& Rotter (1958) was adapted to the present study. It is an 11-point scale numbered 0 through 10 . Printed at the far left of the scale, corresponding to the 0 , was the phrase "Pretty sure light will not come on"; printed at the far right of the scale, corresponding to the 10 , was the phrase "Pretty sure light will come on." Brackets were drawn bounding 5 through 10 (and indicated "yes" responses) and 5 through 0 (and indicated "no" responses). The scale point 5 could be used with either "yes" or "no" and therefore represented the greatest uncertainty of outcome. This scale was placed at the bottom center of the board, thus being easily accessible to Ss.

\section{PROCEDURE}

The Ss were run individually and were told that when the "little" (bottom) light came on, they were to guess whether or not the "big" (top) light would come on. They were told to verbalize either yes or no and then call a number from the scale that "best represented their feelings." After $S$ responded, the big light, if it was scheduled, would come on for about $2 \mathrm{sec}$; then both lights would be turned off together and the next trial would begin. If the light were to remain off, 2 sec after it would have come on the little light was turned off. The intertrial interval approximated 3 to $4 \mathrm{sec}$. Ss received acquisition and extinction training contiguously. In extinction, the big light never came on. An extinction criterion was adopted of eight successive "no" responses of maximum confidence (no-1 or no-0). A maximum of 90 extinction trials was given.

All analyses reported were collapsed over the color variable, since this factor did not influence performance (all Fs $<1$ ).

It will be recalled that the scale point 5 represented maximum uncertainty, and scores on Trial 1 reflected little bias towards expecting one or the other of the outcomes; the means were 5.5, 5.4, and 5.4 for Groups $C, M$, and $F$, respectively (F < 1). Analysis of terminal trial expectancies for each group yielded a significant $F$ ratio, $F(2,27)=71.19$, $p<.01$; means for Groups $C, M$, and $F$ were $9.82,4.44$, and 5.55 , respectively. A Duncan's multiple range test revealed that only the comparisons involving Group C

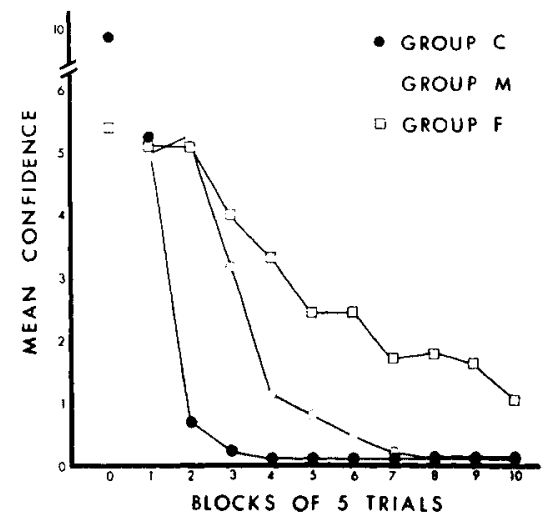

Fig. 1. Mean confidence as a function of terminal acquisition (Point 0 ) and extinction, blocked by five trials for Groups C, M, and F. 
were reliable $(p<.01)$. These means are plotted in the extreme left in Fig. 1.

One measure of extinction performance was the total number of yes responses emitted by Ss. Over the first 30 trials, Groups $C, M$, and $F$ gave means of 3.0,6.8, and 7.6 such responses. A chi-square analysis indicated that response frequencies were not distributed by chance alone $\left(\chi^{2}=19.56\right.$, df $\left.=2, \mathrm{p}<.01\right)$; however, a second analysis demonstrated that the two PR groups were responding "yes" with comparable frequency $\left(\chi^{2}=.44, \mathrm{df}=1\right.$, $\mathrm{p}>$.05).

A second measure of extinction performance was provided by the "expectancy" ratings accompanying the yes and no responses. These scores were blocked by five trials and are presented in Fig. 1. Most of the scores corresponded to "no" responses, especially after the second trial block. Scores closer to zero reflect increasing certainty in the absence of the light. It will be observed that the two PR groups tended to be differentially certain on this confidence measure, whereas they were giving about the same number of no and yes judgments. An analysis of variance indicated that all three experimental sources of variance were significant beyond the $1 \%$ level: trials, $F(9,243)=59.10$; groups, $F(2,27)=17.64$; Trials by Groups, $F(18,243)=5.96$. A Duncan's range test showed that the means of $.64,1.68$, and 2.90 for Groups $\mathrm{C}, \mathrm{M}$, and $\mathrm{F}$ differed significantly $(\mathrm{p}<.01)$ among themselves. Groups $M$ and $F$ were not different during the first 10 trials, but then begin to diverge, Group $F$ being the more resistant of the two.

A third measure of extinction performance, trials to criterion, indicated the same relationship. The means of 5.2, 18.3 , and 43.7 for Groups $C, M$, and $F$, respectively, were significantly different, $F(2,27)=42.61, \quad p<.01$. A Duncan's range test demonstrated that all three groups differed from one another ( $p<.01)$. Ranking them, from most to least resistant to extinction, the groups were $\mathbf{F}, \mathbf{M}$, and $\mathrm{C}$.

\section{DISCUSSION}

The major finding of the present study was that the sequential variable of run length effectively differentiated the $P R$ groups in extinction. This result is analogous to that of N-length manipulations in the animal laboratory (e.g., Capaldi, 1964), and may be understood in an analogous manner. RE can be considered to be a function of the number of -+ transitions, of the length and frequency of run lengths experienced by an $S$, and of the amount of geneneralization of the response tendency to other run lengths. From the mechanics of determining RE that are presented elsewhere (Capaldi, 1964), it is clear that in early extinction a group receiving run lengths of 1 and 4 at frequencies of 5 and 3 , respectively, should be comparable to a group receiving run lengths of $1,2,3$, and 4 at frequencies of $3,2,2$, and 1 , but more resistant during late extinction due to the greater generalization resulting from more concentrated experience at the longer run length.

The present experiment differs from much of the human and animal PR literature in an important respect, namely, that the traditional research has usually dealt with actual rewards and nonrewards. The only reinforcement and nonreinforcement (or mild punishment) stimuli that were ostensibly involved in the present study are those that might be linked to the accuracy of the responses of the Ss. Since the event outcome was unpredictable in each PR group, it is reasonable to assume that both types of responses (positive and negative) were occurring before both - and + events and that each was both "reinforced" (i.e., the guess was correct) and "nonreinforced" (i.e., the guess was incorrect). The quasirandom distribution of response-reinforcement combinations for each $S$ in each PR group would not serve, presumably, to differentiate the groups in extinction. The factor which could, and did, serve this function apparently was differential experience with the sequence of acquisition phase events.

What is comparable for all PR experiments is that the event that constitutes the run length is that event that is to be present during extinction. Thus, Ss may receive runs of nonrewarded trials broken up by rewarded trials, or they may receive runs of "light-off" broken up by "light-on" trials. In either case, however, it is the persistence of the response that anticipates the other event (that which defines the run length) that is taken as the measure of RE. The model presented here is open ended on the status of reinforcement in PR. It might be argued, for example, that a reinforcement event serves two functions, in that it not only strengthens the response that is being measured and about which statements of $\mathrm{RE}$ are made, but also serves to define the various run lengths experienced by $S$. In studies in which reinforcement is used, these two functions are confounded.

\section{REFERENCES}

CAPALDI, E. J. Effect of N-length, number of different N-lengths, and number of reinforcements on resistance to extinction. Journal of Experimental Psychology, 1964, $68,230-239$.

CAPALDI, E. J. Partial reinforcement: An hypothesis of sequential effects. Psychological Review, 1966, 73, 459-477.

GAMBINO, B., \& MYERS, J. L. Role of event runs in probability learning. Psychological Review, 1967, 74, 410-419.

GROSSLIGHT, J. H., \& CHILD, I. L. Persistence as a function of previous experience of failure followed by success. American Journal of Psychology, 1947, 60, 378-387.

GROSSLIGHT, J. H., HALL, J. F., \& MURNIN, J. Patterning effect in partial reinforcement. Journal of Experimental Psychology, 1953, 46, 103-106.

HUMPHREYS, L. G. The effect of random alternation of reinforcement on the acquisition and extinction of conditioned eyelid reactions. Journal of Experimental Psychology, 1939a, 25, 141-158.

HUMPHREYS, L. G. Acquisition and extinction of verbal expectations in a situation analogous to conditioning. Journal of Experimental Psychology, 1939b, 25, 294-301.

JAMES, W. H., \& ROTTER, J. B. Partial and one hundred per cent reinforcement under chance and skill conditions. Journal of Experimental Psychology, 1958, 55, 397-403.

RESTLE, F. Psychology of judgment and choice. New York: Wiley, 1961.

RESTLE, F. Run structure and probability learning: Disproof of Restle's model. Journal of Experimental Psychology, 1966, 72, 383-389.

NOTE

1. This research was supported in part by National Science Foundation Postdoctoral Fellowship No. 48055 to the first author and in part by National Institute of Child Health and Human Development Grant HD 04379 to the second author. 